\title{
Iconografía inédita de la Flora Mexicana, obra de Sessé y Mociño, en el Acervo Histórico del Herbario Nacional de México, MEXU
}

\author{
MARÍA TERESA GERMÁN RAMÍREZ ${ }^{1}$
}

\begin{abstract}
RESUMEN. Se presenta la historia de los dibujos y calcas de plantas, elaborados con base en la colección de Sessé y Mociño, que desde 1897 han existido en la biblioteca de botánica del Instituto de Biología de la UNAM, anteriormente integrada al Instituto Médico Nacional (antecesor del I.B.). Las calcas fueron encuadernadas en 16 volúmenes y fueron elaborados índices para facilitar su consulta.
\end{abstract}

ABSTRACT. A history of the drawings and copies of the original drawings of the plants is presented based on the collections of Mociño and Sessé, kept from 1897 in the botany library of the Instituto de Biologia, UNAM, part of the Instituto Médico Nacional. The copies were bound in 16 volumes and an index was prepared to facilitate consultation.

El conocimiento sobre las colecciones y recolectores de plantas mexicanas, es el punto de partida de trabajos florísticos y taxonómicos, por lo que resulta indispensable conocer itinerarios de las exploraciones efectuadas por los botánicos que nos antecedieron y sus colecciones.

Los nombres de Mariano Mociño y Martín de Sessé miembros de la Expedición Botánica Española, resultan muy comunes para la mayoría de los estudiosos de la flora mexicana. Sin embargo, muy pocos conocen sus colecciones, y menos aún, el sin número de dificultades que vivieron o las consecuencias de éstas, sobre su herbario y manuscritos.

\footnotetext{
'Herbario Nacional, Instituto de Biología, UNAM. Apdo. Postal 70-367, Ciudad Universitaria, Delegación Coyoacán, 04510, México, D.F.
} 
No obstante que hay muchos trabajos publicados acerca de la Real Expedición Botánica Española y biografias de sus integrantes (Ricket, 1947; McVaugh, 1977, 1980), resulta necesario compartir la información aquí incluida, como un apoyo a las investigaciones de la comunidad botánica, ya que estos dibujos sustituyen las muestras de herbario de Mociño y Sessé y en consecuencia, muchos de estos dibujos son considerados los ejemplares TIPO porque fueron la base de especies nuevas, lo cual hace que los estudiosos de la flora mexicana (taxónomos y florísticos) necesiten consultar estos dibujos.

LA ICONOGRAFÍA DE LA FLORA MEXICANA es la culminación de muchos años de trabajo de un equipo de naturalistas enviados a la Nueva España por el rey Carlos III de España en 1787 y algunos mexicanos que se adhirieron a ese grupo, los que excursionaron en territorio mexicano para recolectar muestras botánicas.

Martín de Sessé, director de esta expedición, viajó a España junto con el botánico novohispano José Mariano Mociño con el propósito de publicar su obra, pero la muerte le sorprendió en 1809 recayendo sobre Mociño esa tarea.

La situación política de España impidió a Mociño efectuar la publicación de su obra, teniendo que salir rumbo a Francia al ser expulsado de ese país (con el derrocamiento del gobierno francés en España), ya que fue considerado miembro del gabinete de Napoleón por ocupar el cargo de director del Museo de Historia Natural.

Mociño llego a Montpelllier (ciudad francesa) en 1812, coincidiendo con la estancia de A.P. de Candolle en ese país, entablando inmediatamente relaciones académicas con éste, ya que de Candolle era el director del Jardín Botánico de esa ciudad. Mociño en 1816, ofreció a A.P. de Candolle la colección completa de dibujos y manuscritos, con la condición de que después de haber rectificado la nomenclatura propuesta, insertase en su Prodromus, todas las especies nuevas para la ciencia bajo el nombre de los autores de la Flora de México (obra inconclusa y póstuma de Sessé y Mociño) y descubiertas durante la Real Expedición Científica Española, con la siguiente frase: yo os los doy y os confió para el futuro y el cuidado de mi gloria científica (Colmeiro, 1858).

Tiempo después, A.P. de Candolle salió de Montpellier hacia Ginebra, lugar en donde continuó su obra (Grobet, 1982), en la cual incluyó un número alto de especies mexicanas. Para describir las especies mexicanas se basó esencialmente en los dibujos, ya que además de belleza, tienen detalles de importancia para los taxónomos.

En 1817, Mociño solicitó asilo político a España, a través de la Academia de Medicina de Madrid, consiguiendo asi, el permiso para regresar a ese país. Antes de irse de Montpellier, pidió a A. P. de Candolle la colección de dibujos, ya que consideraba eran propiedad del reino de España y vio la posibilidad de publicar la obra a su regreso. A.P. de Candolle, para evitar quedarse sin material para continuar su Prodromus ya adelantado, pidió ayuda a varios pintores, acudiendo a su llamado casi 
toda la ciudad de Ginebra con dotes pictóricas, obteniendo asi gran cantidad de duplicados de los dibujos (De Candolle, 1874).

A.P. de Candolle organizó los dibujos usados como Tipo para el Prodromus con el nombre de Calques des Dessins de la Flore du Mexique, De Mociño et Sesse dans le Systema ou le Prodromus. Las copias obtenidas las envió a 10 de los primeros museos botánicos (De Candolle, 1874). El resto quedó en varios volúmenes en su herbario, formando la Iconografia Inédita con 110 géneros y 1200 especies nuevas, dejandoles los nombres que Mociño y Cervantes les habían dado (Altamirano, 1898).

Estas dos últimas obras (Iconografia Inédita y Calques.), son conocidas únicamente por unos cuantos botánicos mexicanos. Sin embargo, en varias bibliotecas europeas como las de los herbarios de Kew (K) o Ginebra (G), son obras consultables y cuyo contenido (como se dijo anteriormente) son láminas que sirvieron de ejemplares Tipo a de Candolle, para elaborar el Prodromus. Estas láminas son dibujos a color y quién no conozca que son copias, las toma como originales, error cometido inicialmente por quien escribe ésto, al consultar incidentalmente la colección en la biblioteca del Jardín Botánico de Kew.

La presencia de esas acuarelas en un lugar tan distante, motivó nuestro interés por conocer más sobre la procedencia y autenticidad de éstas, así como la relación con aquellos dibujos polvosos y desordenados revisados superficialmente en México, antes de viajar a Inglaterra. Durante la búsqueda de información y literatura, tuvimos la suerte de rescatar un archivo muerto del Instituto de Biología, en el que fueron localizados documentos referentes a la compra y adquisición de libros y colecciones del Instituto Médico Nacional, institución encargada del estudio de los Recursos Naturales en México a fines del siglo XIX y principios del XX.

En 1896, Fernando Altamirano, director del Instituto Médico Nacional durante su estancia en Europa para asistir a un congreso y a instancias de José Ramírez (investigador del mismo instituto y pionero de la historia de la botánica mexicana), consiguió a través de Casimiro de Candolle, nieto de Alphonso Phyramo (A.P.) una copia de las calcas de esta colección depositada en Ginebra, ya que le fue imposible localizar los dibujos originales que esperaba estuvieran en Madrid (Altamirano, 1898).

La elaboración de las calcas fue supervisada por Casimiro de Candolle en Ginebra, quién no sólo facilitó el juego de holotipos calcados, para los cuáles F. Altamirano envió 300 francos desde París (para pagar a los dibujantes) sino que también ofreció copia de la Iconografía Inédita, asi como copia del prefacio, introducción e írdice (mimeografiado) (fig. 1).

Fernando Altamirano quedó muy agradecido y admirado por la colaboración encontrada en ese notable botánico, heredero de Alphonso Phyramo de Candolle, declarando esto en un discurso pronunciado a su regreso a México (Altamirano, 1898). 
BOLETÍN DE LA SOCIEDAD BOTÁNICA DE MÉXICO, Núm. 54, 1994

Recar da $M^{2}$ If Xamen Alamizano la

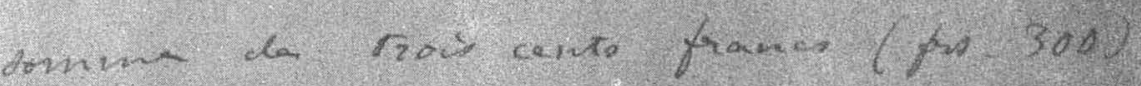

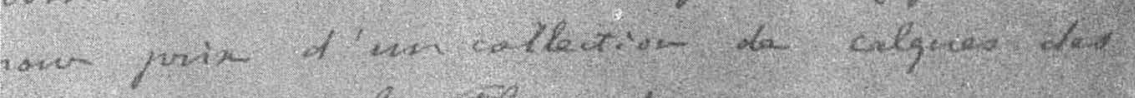

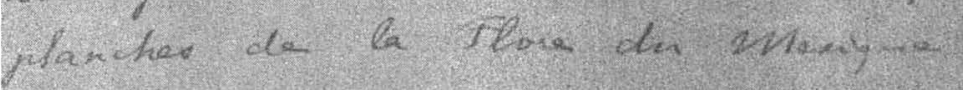

Gonich 2 g nonewwe 1897 $-c \cdot d e$ Canor 100

Batan Atramirano lo

cento francs (yos 300$)$ alective de calgres des

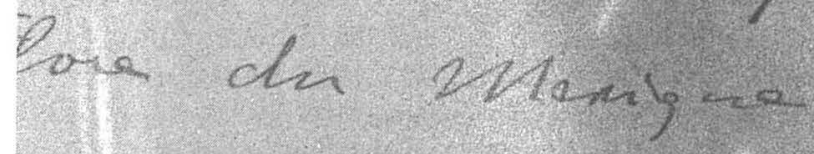

29: nankiva $C \cdot d a c a n d e p l e$

Fig. 1. Recibo de pago por las calcas enviadas a México, firmado por Casimiro de Candolle.

72 
De acuerdo con documentos del Archivo Histórico del Herbario Nacional de México (Fondo Instituto Medico Nacional, Inventarios), el costo de esta colección fue de 514.00 pesos mexicanos (fig.2).

De este juego de calcas copiados directamente de los originales de Ginebra, el dibujante del Instituto Médico Nacional, Adolfo Tenorio, elaboró dibujos a tinta, lápiz y acuarelas a partir de este juego de calcas, para los diferentes proyectos del Instituto Médico Nacional, institución que dió origen al actual Instituto de Biología de la UNAM.

El interés del Instituto Médico Nacional por conseguir documentos históricos, fue motivado por las investigaciones del doctor José Ramírez, quién se encargó de numerar las copias de los dibujos y manuscritos de Sessé y Mociño; escribiendo además algunos artículos sobre las especies mexicanas de estos autores e impulsó la edición de la Flora Mexicana y Plantae Novae Hispaniae (1887, 1a. ed.; 1893, 2a. ed.).

\section{CONTENIDO DE LA OBRA}

La obra que aquí es presentada, es un juego completo de copias o calcas de dibujos de plantas mexicanas denominada: ICONOGRAFIA INÉDITA DE LA FLORA MEXICANA, basada en el juego de láminas a color del herbario de DeCandolle en Ginebra. Estos dibujos se han mantenido en el Acervo Reservado de la Biblioteca del Herbario Nacional, MEXU (conocido anteriormente como Herbario del Instituto Médico Nacional) desde 1897 (fig.1). Esta copia consta de dibujos a lápiz elaborados en papel muy delgado y frágil, cada lámina conserva en el pie de figura el nombre original de la especie y el número de lámina. Algunas de las hojas estan deterioradas pero se conservan bien los dibujos, los cuáles fueron ordenados numéricamente y encuadernados en 16 volumenes, por quién escribe ésto (fig.3).

La ordenación de los dibujos fue numérica, elaborandose un índice para cada volumen; también fue elaborado un índice general por familias, orden alfabético de géneros y volumen. Cada volumen contiene su índice de las especies incluidas en orden numérico.

La colección consta de 1337 dibujos a lápiz de plantas mexicanas y 180 láminas combinadas, teniendo éstas últimas hasta 6 dibujos de diferentes especies por lámina, basados en las colectas efectuadas por la Expedición Real española enviada a México en 1787 y son calcas de las copias de la colección original que recientemente adquirió el Hunt Institute for Botanical Documentation (Jacobson, 1981).

El contenido de la obra es resumido en los cuadros 1 y 2 y la lista de especies en orden alfabético de familias y géneros que es anotada más adelante. 


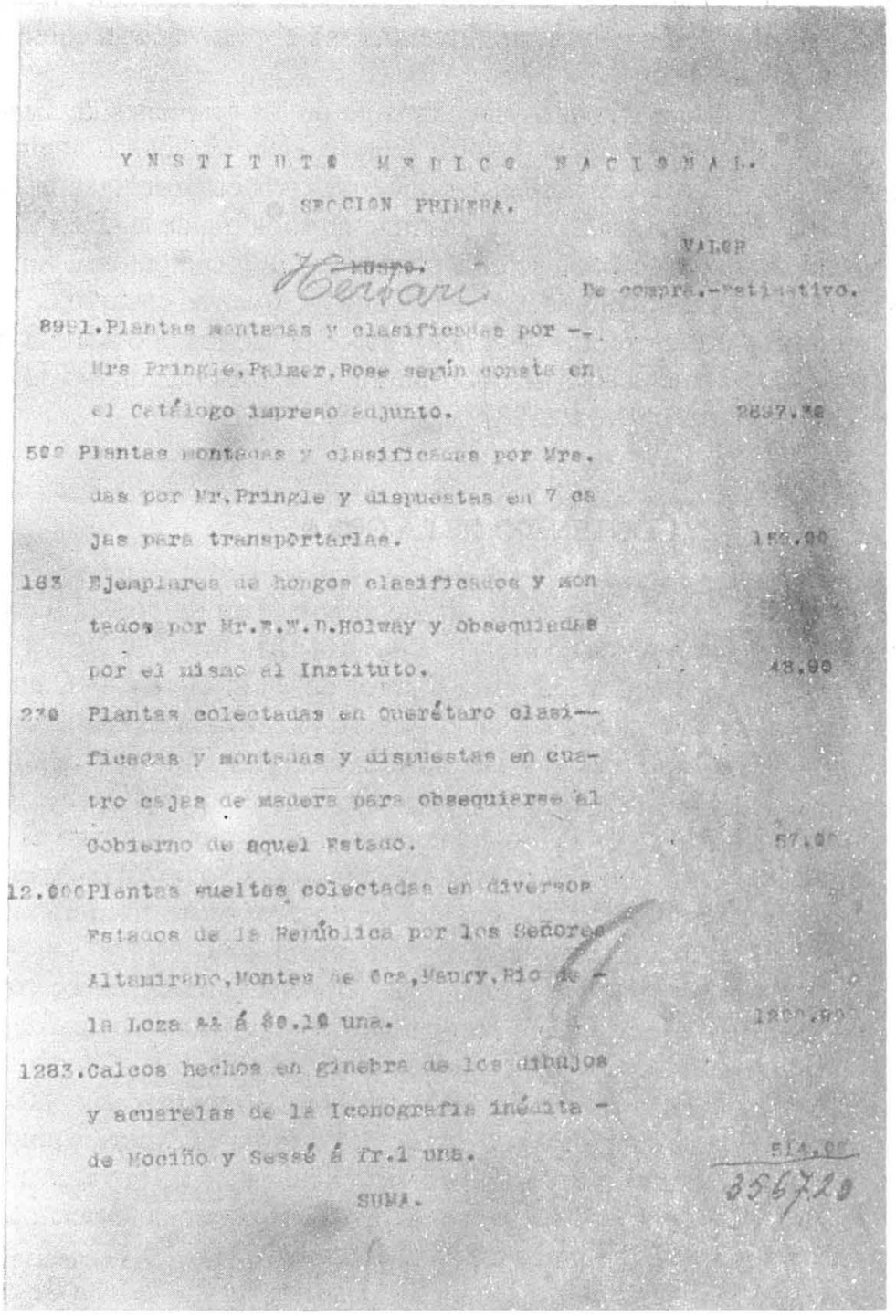

Fig.2. Registro de inventario de los dibujos en el Instituto Médico Nacional. 
ICONOGRAFÍA DE SESSÉ Y MUCIÑO EN ACERVO HISTÓRICO, MEXU

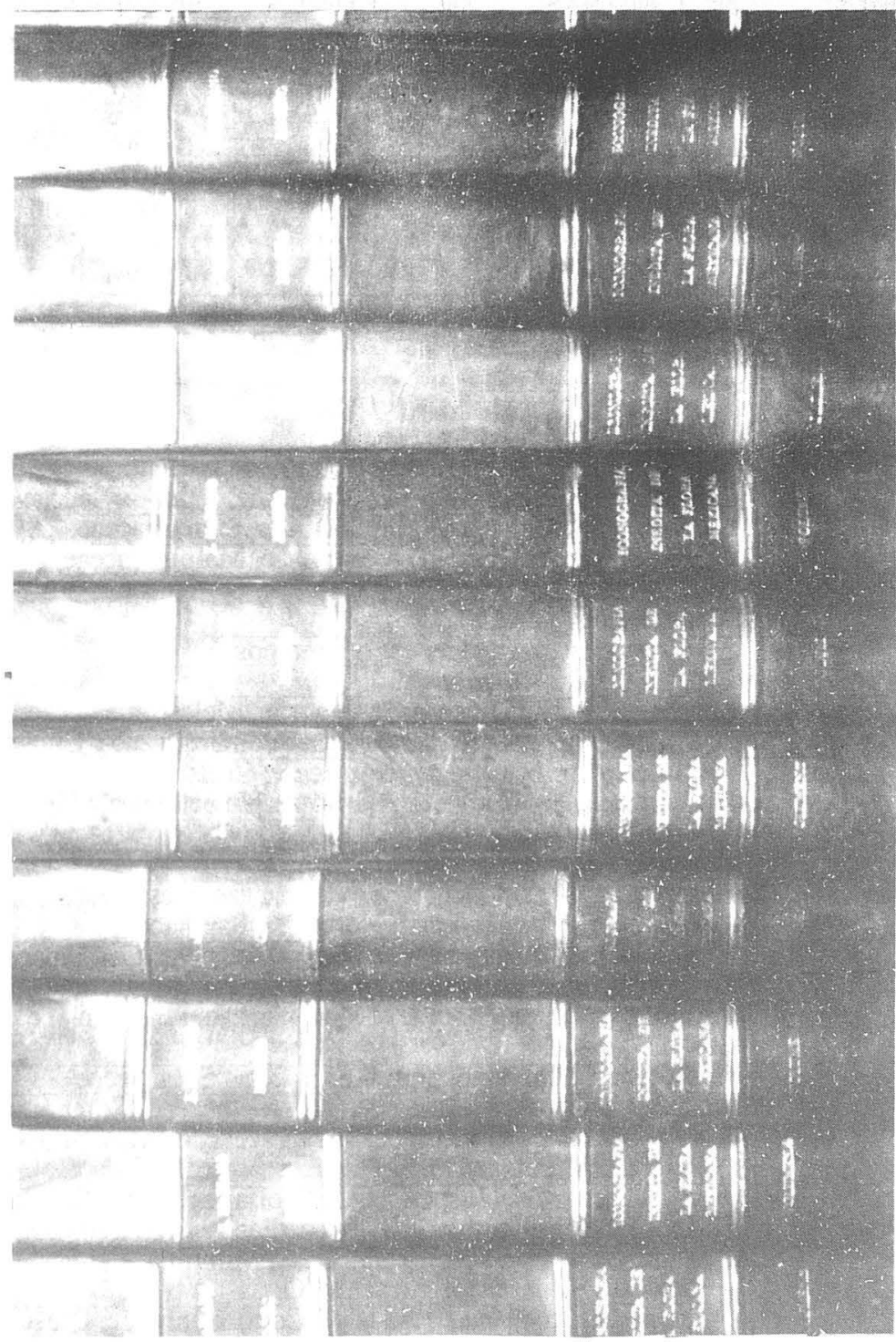

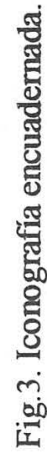


CUADRO 1. Láminas contenidas por volumen.

\begin{tabular}{|c|c|c|c|}
\hline No. VOLUMEN & No. LAMINAS & No. VOLUMEN & No.LAMINAS \\
\hline 1 & $1-66$ & 9 & $601-703$ \\
\hline 2 & $67-123$ & 10 & $704-804$ \\
\hline 3 & $124-185$ & 11 & $805-905$ \\
\hline 4 & $186-250$ & 12 & $906-1015$ \\
\hline 5 & $251-313$ & 13 & $1016-1108$ \\
\hline 6 & $314-385$ & 14 & $1109-1200$ \\
\hline 7 & $386-507$ & 15 & $1201-1300$ \\
\hline 8 & $508-600$ & 16 & $1301-1337$ \\
\hline
\end{tabular}

CUADRO 2. Familias contenidas por volumen.

\begin{tabular}{|c|c|}
\hline No.VOL & FAMILIAS \\
\hline 1 & $\begin{array}{l}\text { Berberidaceae, Capparidaceae, Caryophyllaceae, Cistaceae, Cruciferae } \\
\text { Magnoliaceae, Malvaceae, Menispermaceae, Papaveraceae, Passifloraceae, } \\
\text { Polygalaceae, Ranunculaceae, Violaceae, Zanthoxylaceae }\end{array}$ \\
\hline 2 & Bytneriaceae, Malvaceae, Sapindaceae, Sterculiaceae, Tiliaceae \\
\hline 3 & $\begin{array}{l}\text { Geraniaceae, Guttiferae, Hypericaceae, Malphigiaceae, Meliaceae, } \\
\text { Oxalidaceae, Rutaceae, Flacourtiaceae, Samydeae, } \\
\text { Rhamnaceae (Trangulaceae) }\end{array}$ \\
\hline 4 & Anacardiaceae, Caricaceae y Leguminosae \\
\hline 5 & Leguminosae, Rosaceae \\
\hline 6 & $\begin{array}{l}\text { Loasaceae, Rizophoraceae, Lythraceae, Melastomataceae, } \\
\text { Myrtaceae, Onagraceae, Turneraceae, Combretaceae y Cucurbitaceae }\end{array}$ \\
\hline 7 & $\begin{array}{l}\text { Araliaceae, Capprifoliaceae, Cornaceae, Crassulaceae, Saxifragaceae, } \\
\text { Loranthaceae, Portulacaceae, Rubiaceae, Umbelliferae y Valerianaceae }\end{array}$ \\
\hline 8 & Compositae \\
\hline 9 & Compositae \\
\hline 10 & $\begin{array}{l}\text { Apocy naceae, Campanulaceae, Ebenaceae, Gesneriaceae, } \\
\text { Lobeliaceae, Myrsinaceae, Ericaceae y Sapotaceae }\end{array}$ \\
\hline 11 & $\begin{array}{l}\text { Bignoniaceae, Borraginaceae, Convolvulaceae, Gentianaceae, } \\
\text { Hydrophyllaceae, Liliaceae, (Bromeliaceae, Amarylidaceae, } \\
\text { Agavaceae, Pontederiaceae), Martyniaceae, Polemoniaceae y } \\
\text { Scrophulariaceae }\end{array}$ \\
\hline 12 & Besleriaceae, Labiatae, Scrophulariaceae, Solanaceae \\
\hline 13 & $\begin{array}{l}\text { Acanthaceae, Amaranthaceae, Aristolochiaceae, Combretaceae, } \\
\text { Chenopodiaceae, Elaeagnaceae, Lauraceae, Lentibulariaceae, } \\
\text { Nyctaginaceae, Polygonaceae, Plumbaginaceae, Primulaceae, Pirenaceae }\end{array}$ \\
\hline 14 & $\begin{array}{l}\text { Bignoniaceae, Cochlospermaceae, Coriariaceae, Cycadaceae, } \\
\text { Dilleniaceae, Euphorbiaceae, Eriocaulaceae, Fumariaceae, } \\
\text { Leguminosae, Loasaceae, Malvaceae, Monimiaceae, } \\
\text { Pirolaceae, Sabiaceae, Urticaceae, Otras }\end{array}$ \\
\hline 15 & $\begin{array}{l}\text { Alismaceae, Anonaceae, Araceae, Commelinaceae, Dioscoreaceae, } \\
\text { Juncaceae, Iridaceae, Musaceae, Orchidaceae }\end{array}$ \\
\hline 16 & Compositae, Gramineae, Pteridophytae, Otras \\
\hline
\end{tabular}




\section{COMENTARIOS}

La obra que aquí presentamos ha sido totalmente desconocida en México, tal vez, debido a la condición en la que estos dibujos se encontraron por casi un siglo, los que, desordenados y casi imposible de consultarse han sido de poca útilidad. Sin embargo, a partir de su encuadernación, son muchos los especialistas que los consultan, pero al desconocer su procedencia y autenticidad existe inseguridad en su uso, razon que nos ha llevado a escribir este documento.

Nuestro interés en la obra, surgió básicamente de ver que una colección tan importante estuviera deteriorandose, por lo que fuimos ordenando los dibujos numéricamente, hasta lograr su arreglo en volumenes. Para esta edición, respetamos la nomenclatura genérica y específica así como la numeración de las láminas original, para evitar mayor confusión taxonómica o nomenclatural. Nos permitimos actualizar los nombres de familias obsoletos para facilitar su consulta. Es pertinente señalar, que algunas láminas no aparecen citadas por no localizarse en esta colección.

Agradecimientos. Agradezco al Lic. Armando Butanda su amabilidad por la revisión del manuscrito y sus comentarios, a la Pas. de Biól. Norma Moreno, su ayuda en la mecanografía y al M. en C. Luis M. Pinzón, las facilidades brindadas con el equipo de cómputo.

\section{LITERATURA CITADA}

Altamirano, F., 1898. Adquisiciones de documentos antiguos importantes para el estudio de nuestra flora. Anu. Acad. Mex. Cienc. Ex. Fis. Nat. 4:17-39.

Candolle, A. P. DE, 1874. Calques des Dessins de la Flore du Mexique, de Mociño et Sesse qui ont servi de types d' Especies dans le Systema ou le Prodromus. 6 p.

CANDolle, A. P. DE, Iconografía Inédita de la Flora Mexicana. En: Catalogue des dessins et Descriptions D'Especes de la Flore du Mexique. pp. 55.

Colmeiro, M. 1858. La botánica y los botánicos de la Península HispanoL uisitánica. Madrid. pp. 216.

Grobet, R. 1982. El Peregrinar de las Flores Mexicanas. C. E. C. S. A. México. pp. 100. INVENTARIOS.1909. Archivo histórico I.B. UNAM. Fondo Instituto Médico Nacional.

JACOBSON, T. D. 1981. Longlost Sesse and Mociño illustrations acquired. Bull. Hunt Inst. Bot. Document. 3(1):13.

Mcvaugh, R. 1977. Botanical Results of the Sesse and Mociño Expedition (1787-1803). Contr. Univ. Michigan Herb. 11 (3):97-195.

Mcvaugh, R. 1980. Botanical Results of the Sessé \& Mociño Expedition (1787-1803).II. The Icones Florae Mexicanae. Univ. Michigan Herb. 14: 99-140.

RickeTt,H.W. 1947. The Royal Botanical Expedition to New Spain. Chron. Bot. 11: 1-86. 
Lista de familias y especies representadas en los dibujos

\begin{tabular}{|c|c|c|c|c|c|}
\hline NOMBRE & JUM.LAM. & VOL. & NOMBRE NUN & M.LAM. & VOL. \\
\hline ACANTHACEAE & & & AMARANTHACEAE & & \\
\hline Albatenia fruticosa & 1070 & 13 & Celosia digyna & $1091 *$ & 13 \\
\hline Barberia tetracantha & 1038 & 13 & Celosia dioica & 1091 & 13 \\
\hline Dianthera repens & 184 & 14 & Cerdia purpurascens DC. & 1092 & 13 \\
\hline Dicliptera acutifolia & 1042 & 13 & Iresine celosioides $\mathrm{L}$. & 1091 & 13 \\
\hline Justicia exilis & 1066 & 13 & Gomfrena diffusa & 1093 & 13 \\
\hline Justicia fuperba & 1044 & 13 & Amaryllis ecuestris Ait. & $1239 *$ & 15 \\
\hline Justicia micranta & 1043 & 13 & Amaryllis linearifolia & 1239 & 15 \\
\hline Justicia oxyphylla & 1068 & 13 & AMENTACEAE $=$ ULMA & MEAE & \\
\hline Justicia papilionacea $\mathrm{N}$. & 1064 & 13 & Celtis & 1162 & 14 \\
\hline Justicia phlomoides & 1051 & 13 & AMONIEAE $=$ ZINGIBEF & RACEAE & \\
\hline Justicia polygonoides & 1055 & 13 & Amomum bicolor & 1227 & 15 \\
\hline Justitia & 1042 & 13 & Amomum rutilans & 1226 & 15 \\
\hline Justitia acutiloba & 1059 & 13 & ANNONACEAE & & \\
\hline Justitia appendiculata & 1050 & 13 & Anona fruticosa & 1184 & 14 \\
\hline Justitia arborea & 1063 & 13 & APOCYNEAE $=$ APOCYN & NACEAE & \\
\hline Justitia cardinalis & 1069 & 13 & Apocynum amoenum & 788 & 10 \\
\hline Justitia cordata & 1053 & 13 & Apocynum truncatum & 787 & 10 \\
\hline Justitia chelonoides & 1052 & 13 & Asclepias acuminata & 783 & 10 \\
\hline Justitia ebracteata & 1056 & 13 & Asclepias dodecathea & 782 & 10 \\
\hline Justitia horminoides & 1049 & 13 & Asclepias grandiflora & 785 & 10 \\
\hline Justitia longiflora & 1058 & 13 & Asclepias penduliflora & 785 & 10 \\
\hline Justitia opathacea & 1057 & 13 & Asclepias tuberastrum & 781 & 10 \\
\hline Justitia phoenicea & 1048 & 13 & Asclepias virgata & 784 & 10 \\
\hline Justitia primulina & 1054 & 13 & Cameraria acutiflora & 791 & 10 \\
\hline Justitia pulchella & 1067 & 13 & Cerbera cuneifolia & 793 & 10 \\
\hline Justitia punicea & 1046 & 13 & Cerbera tevethia $\mathrm{L}$. & 792 & 10 \\
\hline Justitia quadrifida & 1062 & 13 & Cerbera ycotli & $792 *$ & 10 \\
\hline Justitia quadrifolia & 1060 & 13 & Cynanchum acuminatum & 773 & 10 \\
\hline Justitia revoluta & 1047 & 13 & Cynanchum oblongifolium & 776 & 10 \\
\hline Justitia tripilistachia & 1065 & 13 & Cynanchum ovatifolium & 777 & 10 \\
\hline Justitia velutina & 1061 & 13 & Cynanchum prostratum & 774 & 10 \\
\hline Justitia venusta & 1045 & 13 & Cynanchum proliferum & 775 & 10 \\
\hline Ruellia aggregata & 1041 & 13 & Echites cordata DC. & 796 & 10 \\
\hline Ruellia ascendens & 1040 & 13 & Echites hypoleuca & 794 & 10 \\
\hline Ruellia fruticosa & 1070 & 13 & Echites uniflora & 794 & 10 \\
\hline Ruellia viscosa & 1039 & 13 & Echites secundiflora DC. & 798 & 10 \\
\hline ALISMACEAE & & & Eurybia stapelioflora & 804 & 10 \\
\hline Alisma verticillata & $1282 *$ & 15 & Gothofreda flavescens & 778 & 10 \\
\hline Sagittaria teretifolia & 1282 & 15 & Gothofreda periploca & 779 & 10 \\
\hline
\end{tabular}


Haemadictyon

mexicanum DC.

Pergularia albida

Pergularia carnea

Plumieria emarginata

Plumieria multiflora

Plumieria muricata

Plumieria sanguinea

Tabernaemontana alba

Tabernaemontana lutea

Vallesia flavescens

ARACEAE

Caladium pedatum

Caladium virens

Photos scolopendria

Photos stipulata

Thermogeton flagellare

ARALIACEAE

Aralia acutifolia

Aralia alismofolia

Aralia chilapensis

Aralia cymbigera

Aralila decaphylla

Aralia fasciculata

Aralia geminiflora

Aralia oxyphylla

Aralia spinosa

Aralia tomentosa

.Johrenia araliastrum

ARISTOLOCHIAE = ARISTOLOCHIACEAE

Aristolochia genuflora 1108

Aristolochiamexicana 1107

Aristolochia

$$
\text { odoratissima } \mathrm{L} \text {. }
$$

1108

BERBERIDEAE=BERBERIDACEAE

Berberis pinnata $\mathrm{DC}$.

13

BESLERIAE

Alloplectus glaber DC.

932

Besleria ascycardia

931

930

Besleria floresciuri

Besleria resupinasa

929

Herpestis flava

934

10

10

10

10

15

15

15

15

15

436

432

434

433

439

437

431

438

$433 *$

440

13

13

13

7

7

7

7

\section{7}

\section{7}

7

7

\section{7}

7
Herpestis monniera

935

10

10

10

10

10

10

Herpestis pentandra
BIGNONIACEAE

933

12

Amphilophium mutisi

Badiera cirrhosa

DC. 828

828

Badiera conjugata

829

Bignonia brachystemon

826

Bignonia brevisiliqua

827

Bignonia coleoflora

821

Bignonia kerese

822

Bignonia martynioides

Bignonia muricata

823

820

Bignonia nerioides

825

822

Bignonia tubaeformis

824

Bignonia vertiicillata

Chilopsis linearis

825

Crescentia cufetioides

1184

Crescentia cufetioides

1185

830

Parmentiera edulis DC.

Pithecoctenium

$$
\text { muricatum DC. }
$$

820

Tecoma fuscata DC.

823

BORRAGINEAE=BORAGINACEAE

Antiphytum mexicanum DC. 901

Cordia aggregata

879

Cordia crispiflora DC.

875

Cordia dodecandra DC.

874

Cordia linearis

883

Cordia longistyla

876

Cordia nodosa LAM.

882

Cordia parviflora

878

881

Cordia parviflora

Cordia pauciflora

877

Cordia petiolaris

878

Cordia rotata DC.

Cordia tenuiflora

880

876

Cordia ulmifolia

Echium longiflorum

891

903

Ehretia angustifolia

883

Ehretia ceanothina

890

Ehretia cordifolia

884

889

886
11

\section{1}

11

11

11

11

11

11

11

11

11

11

11

11

14

14

11

11 


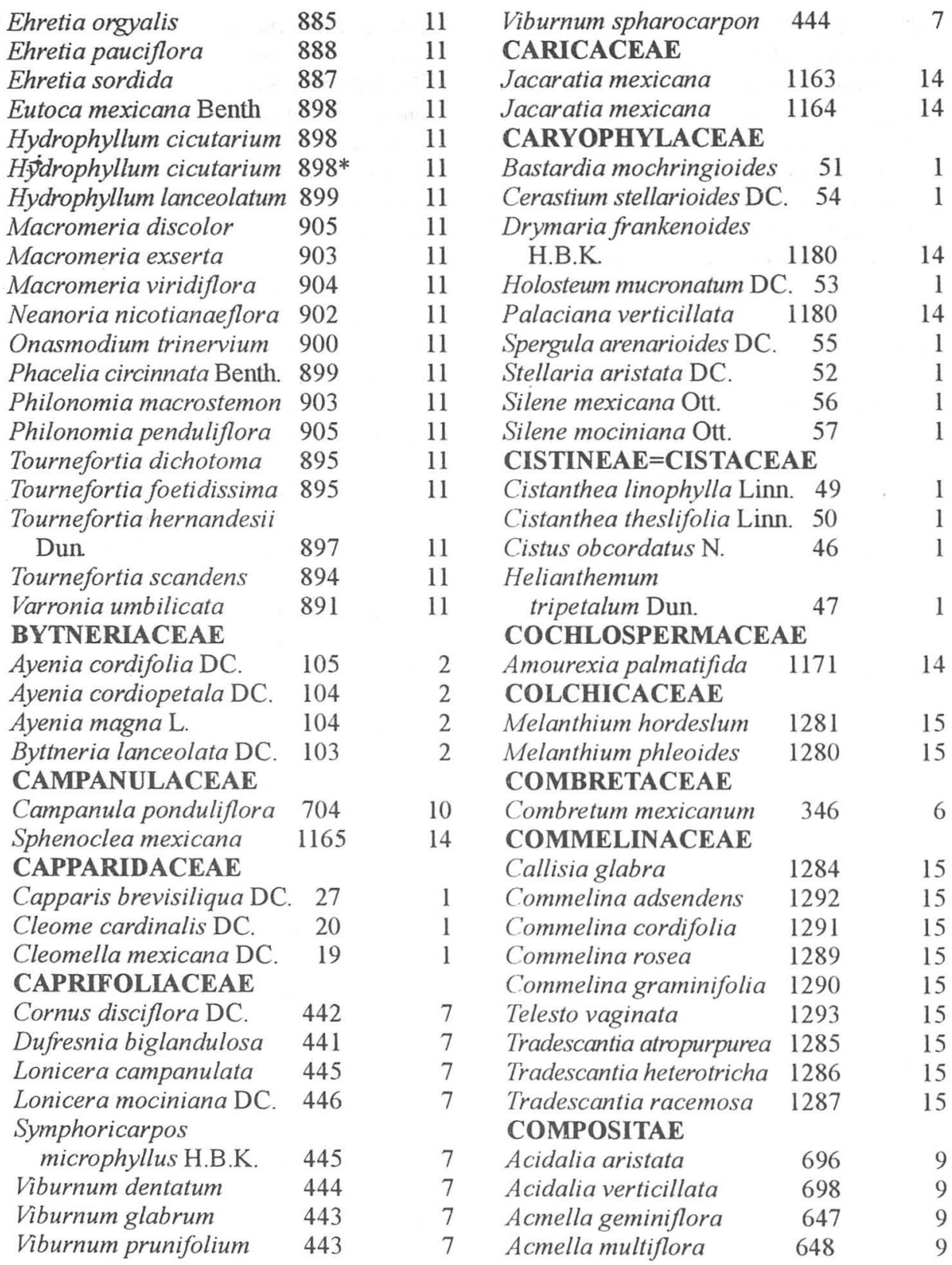


Acourtia formosa DC. $\quad 516$ Acourtia hebeclaua DC. 512 Ageratum purpureum N. 549 Ageratum viscosum N. $\quad 550$ Actinomeris tetragona DC. 603 Actinaceae integrifolia 575 Actinaceae multifida $\quad 574$ Adenophyllum capillaceum DC. Adenotis anthemoides 620 Adenotis oxyphylla 692 690 Adenotis tenuiflora 691 Aganippea bellidiflora DC. 700 Ageratum erigerioides 559 Ageratum thyrsoideum DC. 561 Amellus anthemoides Amellus chrysanthus Amellus parviflorus Amellus polyanthos Amellus trinervis Amellus zinniaflorus Ambrosia absinthiifolia Ambrosia arthemilifolia Linn.

Ambrosia lycopifolia Ambrosia parthenifolia Anaxabia glabra

Anaxabia pubescens Anthosphaera budleioides Aster

Aster chinensis L.

Aster mexicanus

Baccharis diversiflora

Bidens funiculifolia DC.

Bidens helianthoides H.B.K. 610

Bidens purpurea DC.

Boebera alternifolia

Boebera grandiflora

Bulbostylis cavanillesii DC. 566

Bulbostylis veronicaefolia 564

Cacalia acuminata

Cacalia nutans $\mathrm{N}$.

Cacalia pinnatifida
641

640

643

644

642

645

639

639

637

638

669

668

685

703

588*

588

569

611

608

636

635

\section{1}

524

525
8

8

8

8

9

8

8$$
9
$$

9

9

9

9

9

\section{8}

8

9

9

9

9

9

9

9

9

9

\section{9}

9

(1)

9

9

9

9

9

9

8

8

8

8

9

9

9

列

8

8

8

8

8

$\begin{array}{lll}\text { Cacalia simuata Lalc. et Lex. } & 522 & 8 \\ \text { Cacalia tussilaginea } & 524 & 8 \\ \text { Calais multifida } & 689 & 9 \\ \text { Carduus mexicanus } & 508 & 8 \\ \text { Carminatia tenuiflora DC. } & 665 & 9 \\ \text { Cartheuseria pedunculata } & 693 & 9 \\ \text { Centaurea carthamoides } & 509 & 8 \\ \text { Centaurea patens } & 511 & 8 \\ \text { Cineraria ambigua } & 576 & 8 \\ \text { Cineraria } & & \\ \quad \text { dracunculoides Moc. } & 578 & 8 \\ \text { Cineraria malopoides } & 580 & 8 \\ \text { Cineraria mutans } & 582 & 8 \\ \text { Cineraria parviflora } & 577 & 8 \\ \text { Cineraria praecox Cav. } & 578 * & 8 \\ \text { Cineraria salicifolia } & 579 & 8 \\ \text { Clanijeria trifida } & 675 & 9 \\ \text { Clavijeria varifolia } & 674 & 9 \\ \text { Cnicus benedicta L. } & 509 & 8\end{array}$

Coreopsis

artemisiaefolia Jacq. $615 \quad 9$

Coreopsis chaerophyllea $613 \quad 9$

Coreopsis cosmos DC. 615* 9

Coreopsis georgina $\quad 618 \quad 9$

Coreopsis daucifolia $\quad 612 \quad 9$

Coreopsis leucanthemoides $617 \quad 9$

Coreopsis variifolia $616 \quad 9$

$\begin{array}{lll}\text { Coreopsis tuberosa } & 614 & 9\end{array}$

$\begin{array}{lll}\text { Conyza secunda } & 558 & 8\end{array}$

Coreopsis atrorubens $\quad 605 \quad 9$

Cineraria barbarefolia $581 \quad 8$

Coreopsis erythrophaea $606 \quad 9$

Coreopsis foeniculacea $611 \quad 9$

Coreopsis millefolia $\quad 609 \quad 9$

Coreopsis purpurea $\quad 607 \quad 9$

Coreopsis serrata $\quad 610 \quad 9$

Coreopsis tuberosa $\quad 614$

Coreopsis variifolia $\quad 616 \quad 9$

Cosmos sulfureus Cav $615 \quad 9$

Costaea parviflora $671 \quad 9$

Chlamysperma pratense L. 68899

Dysodia grandiflora DC. 6359

Dysodia porophylla B.Lag. 6369 
Erigeron rubricaule $\quad 634$

Erycine oppositifolia

699

Eryocephalus

$$
\text { arthemisifolius }
$$

703

Eupatorium axillare Moc 566

Eupatorium

calamintaefolium

562

Eupatorium

glechomofolium

564

Eupatorium petiolare DC. 567

Eupatorium quaternatum 563

Eupatorium

squarrosum Cav.

566

Eupatorium tenuiflorum 568

Eupatorium thyrsoideom 561

Eupatorium uniflorum

Eupatorium viburnoides

Euphrosine

parthenifolia DC.

526

565

Flaveria angustifolia Pers. 594

Flaveria angustifolia Pers. 595

Flaveria contrayerba

593

Flaveria gymnostyles

Flaveria integrifolia

593

594

595

Flanseria ambrosioides

656*

Franseria xanthioides

656

Florestina tripteris DC. $\quad 528$

Galeana hastata Lall.

688

Georgina acutiflora

Grindelia serrata

554

598

688

Gymnotis micrantha

590

Gymnostiles parthenifolia

Gymnostilis pedunculata

589

Helianthus altissimus L.

Helianthus hirtus

600

620

Helianthus peduncularis

Helianthus purpureus

601

602

603

Heterospermum hybridum 623

Hilairia paniculata

518

Hunteria columbina

520

Inula leptorrhiza
9 Inula longifolia

651

$9 \quad$ Isodorea centauroides $\quad 702$

Isodorea jaceoides

703

$9 \quad$ Isotypus onoseroides

8 Kunth.

518

Klemia glandulosa

557

8

Lagascea mociniana DC. 571

Lemerya anthemoides $\quad 657$

8

8

Lepidopappus tripteris

528

670

694

695

Marumia aurantiaca

Melampodium adscendens 627

8

8

8

8

8

Melampodium connatum 631

Melampodium divaricatum 629B

Melampodium gracile $\quad 626$

Melampodium

multiradiatum

633

8

Melampodium octoradiatum

632

8 Melampodium

8

8

8

8

8

9

9

8

9

8

8

9

8

8

8

8

9

9

9

9

8

8

9

\section{perfoliatum Kunth}

Melampodium pygnidum

631

629

Melampodium

$$
\text { sessiliflorum }
$$

Melanthera angustifolia

552

Mikania alternifolia

556

Mikania ciliata

555

Milleria dyplantha

591

Milleria villosa

592

Monosis salicifolia DC. $\quad 526$

Montaynda frutescens 677

Montagnea tomentosa Lall. 683

Montaynda grandiflora DC. 679

Oligotes biaristata

687

Oligotes triaristata

686

Pectis prostrata Cav.

703

Perdicium cordatum $\mathrm{N}$.

512

Perezia fruticosa

516

517

527

Phania trinervia DC.

551

Piqueria trinervia Cov.

Porophyllum coloratum DC. 519 


\begin{tabular}{|c|c|c|c|c|}
\hline Porophyllum linaria & 520 & 8 & Stevia febrifuga & 551 \\
\hline Porophyllum & & & Stevia glabra & 531 \\
\hline macrocephalum DC. & 557 & 8 & Stevia hymenopappa & 530 \\
\hline Priestleya controversa & 684 & 9 & Stevia lanceolata & 532 \\
\hline Priestleya corymbosa & 683 & 9 & Stevia laxiflora DC. & 540 \\
\hline Priestleya enneantha & 681 & 9 & Stevia leuconeura DC. & 546 \\
\hline Priestleya longifolia & 679 & 9 & Stevia longifolia & 538 \\
\hline Priestleya paniculata & 682 & 9 & Stevia longifolia & $\mathrm{XX}$ \\
\hline Priestleya pinnatifida & 678 & 9 & Stevia longiseta & 546 \\
\hline Priestleya squarrosa & 677 & 9 & Stevia lymenopappa & $\mathrm{XX}$ \\
\hline Priestleya trichotoma & 680 & 9 & Stevia lucida Lag. & 530 \\
\hline Proustia reticulata Jacq. & 514 & 8 & Stevia micrantha Lag. & 548 \\
\hline Pteronia porophyllum Cav & v. 636 & 9 & Stevia multifida DC. & 547 \\
\hline Rea acuminata & 1181 & 14 & Stevia nepetella & 542 \\
\hline Rudbeckia auriculata & 654 & 9 & Stevia nepetafolia Kunth & 543 \\
\hline Rudbeskia diversicolor & 703 & 9 & Stevia ovata WD. & $541^{*}$ \\
\hline Salmea alternifolia & 649 & 9 & Stevia paniculata & 541 \\
\hline Sanvitalia procumbens & $624^{*}$ & 9 & Stevia pubescens & 543 \\
\hline Sanvitalia silphicides & 624 & 9 & Stevia purpurea Pers. & $540 *$ \\
\hline Schkuhria erigeroides & 621 & 9 & Stevia purpurascens & 536 \\
\hline Senecio amplexicaulis & 587 & 8 & Stevia pycnanthema & 534 \\
\hline Senecio aurantiacus & 583 & 8 & Stevia subpurpurascens & 532 \\
\hline Senecio barebjohannis DC & C.577 & 8 & Stevia trachelioides & 533 \\
\hline Senecio linearis & 586 & 8 & Stevia trachelioides & $\mathrm{XX}$ \\
\hline Senecio pinifolius & 585 & 8 & Stevia tripaleaceae & 537 \\
\hline Senecio salignus DC. & 578 & 8 & Stevia tripaleaceae & $X X$ \\
\hline Senecio sonchoides & 584 & 8 & Stevia villosa & 549 \\
\hline Senecio vernus DC. & 579 & 8 & Stevia viminea Schrad. DC. & 538 \\
\hline Shawia corymbosa & 526 & 8 & Stevia viscida Kunth & 549 \\
\hline Siegesbeckia integrifolia & 653 & 9 & Tithonia tagetiflora & 655 \\
\hline Siegesbeckia serratifolia & 652 & 9 & Trimetra ficoidea $\mathrm{DC}$. & 676 \\
\hline Solidago mexicana L. & $703 \mathrm{E}$ & 9 & Tristania fuscata & 519 \\
\hline Soliva mexicana DC. & 589 & 8 & Trixis bracteolata & 513 \\
\hline Spallankania albiflora & 662 & 9 & Trixis cordata & 513 \\
\hline Spallankania flava & 664 & 9 & Trixis corymbosa & 516 \\
\hline Spallankania purpurescen & ss 663 & 9 & Trixis decurrens $\mathrm{DC}$. & 515 \\
\hline Spilanthes $s p$ & 693 & 9 & Trixis liabridis & 517 \\
\hline Stevia acuminata & 535 & 8 & Trixis ochroleuca & 514 \\
\hline Stevia acuminata & $X X$ & 16 & Verbesina alata Lin. & $595^{*}$ \\
\hline Stevia alternans & 545 & 8 & Verbesine oppositifolia & 673 \\
\hline Stevia calaminthae & 544 & 8 & Verbesina pterocaula DC. & 597 \\
\hline Stevia connata & 531 & 8 & Verbesina virgata var. & \\
\hline Stevia eupatoria & 540 & 8 & conyzoides & 596 \\
\hline
\end{tabular}


Vernonia schiedeana Less. 558 Woodvillea incrassata 658 Werneria mociniana DC. 667 Zinnia bicuspis DC. $\quad 573$

Zinnia parviflora

CONVOLVULACEAE

Cobaea acutiflora 572

Convolvulus arboreus

584

Convolvulus brachygonius 841

Convolvulus brevipes $\quad 843$

Convolvulus cirrhosus $\quad 848$

Convolvulus coeruleus 1188

Convolvulus dentatifolius 846

Convolvulus denticulatus 842

Convolvulus eriospermus 847

Convolvulus lividus

Convolvulus longiflorus

Convolvulus nivens

Evolvulus cordifolius

Evolvulus renifolius

Evolvulus tenuifolius

Exogonium corymbosum

Exogonium cumflorum

Exogonium umbellatum

Exogonum eriospermun

Choisy

Ipomaea nutans Choisy

Ipomaea bracteata

Ipomaea bulbocastana

Ipomaea digitata

Ipomaea gracilis

Ipomaea erythraea

Ipomoea humilis

Ipomoea mexicana

Ipomoea multiflora

Ipomoea temocoatl

Pharbitis heterophylla

Quamoclit coccinea

$$
\text { Maench. }
$$

Quamoclit hederifolia

Quamoclit vitifolia Don

849

844

845

865

864

866

862

863

861

847

860

854

857

859

852

858

856

853

851

855

857

858

863

861

CORARIACEAE

Coriaria atropurpurea DC. 1167
8

8

11

11

11

11

11

14

11

11

11

11

11

11

11

11

11

11

11

11

11

11

11

11

11

11

11

11

11

11

11

11

11

11

11

14
CRUCIFERAE

Nasturtium mexicanum DC. 18

CUCURBITACEAE

Momordica anguria

$347 *$

Momordica charantia

$347 *$

Sechium palmatum DC. 355

CYCADACEAE

Zamia integersima

1185

14

CYNOMORIACEAE

Cynomorium mexicanum $1166 \quad 14$

EBENACEAE

Symplocos ebracteata $\quad 733 \quad 10$

Symplocos ferruginea $\quad 734 \quad 10$

CRASSULACEAE

Nitella alternifolia

421

418

7

Sedum arbuscula

CHENOPODEAE $=$ CHENOPODIACEAE

7

Petiveria ochroleuca Moq. $1094 \quad 13$

Rivina mexicana Moq. $1095 \quad 13$

DILLENIACEAE

Leucothea terniflora

Tetracera erecta DC.

1179

14

DIOSCOREACEAE

$\begin{array}{lll}\text { Dioscorea hiandra } & 1274 & 15 \\ \text { Dioscorea sativa } & 1274 & 15 \\ \text { Dioscorea triandra } & 1274 & 15\end{array}$

ELOEAGNEAE $=$ ELAEAGNACEAE

Bucida spinosa

1105

13

Durandea pendula

1106

13

EUPHORBIACEAE

Acalypha heterostachya

1111

14

Acalypha pastoris

1112

Androstachys lanceolata 1122

14

Androstachys rhombifolia 1121

14

Croton acuminatum

1117

('roton cardifolium

1113

('roton gracile

1116

('roton laxiflorum

1119

('roton oxyphyllum

1115

('roton penicillatum

$1120 *$

('roton pubiflorum

1118

('roton verticillatum

1114

14

14

14

14

14

14

14

14

Croton vulpinus

1116 
Dalambertia lanceolata Mull.

Dalambertia populifolia Baill. Euphorbia ciliatiflora Euphorbia cyathophora Euphorbia edulis Euphorbia peltata Euphorbia quinquefolia Euphorbia scaposa Euphorbia splendidissima 1145 Euphorbia tetlatzian $\quad 1146$ Euphorbia trifolia $\quad 1142$

Gymnostillingia macrantha Mull.

1123

Hura polyandra Baill.

Iphys fasciculata

1128

1176

Jatropha gossypiifolia

1129

Jatropha grandiflora

1130

Jatropa janispha

1133

Jatropha jarquiniana ovata 1130

Jatropha pseudocurcas

Jatropha tuberosa

Jatropha triloba

1131

Malaspina volubilis

Manihot foetida

Manihot steniloba

Pedilanthus aphyleus

Pedilanthus bracteatus

Pedilanthus nutans

Phyllanthus arboreus

Phyllanthus ovatifolius

Physocarpum multiflorum

Plukenctia integrifolia

Sapium brachystachyum

Sapium eglandulatum

Sapium macradenum

Sapium persicaefolium

Stillingia suffruticosa

Tricera ovata

Xylophylla mexicana

ERIOCAULACEAE

Eriocaulon anesps
1132

1134

1136

1134

1133

1150

1149

1148

1110

1109

1135

1138

1126

1127

1124

1125

1123

1137

1137*

1184

\section{FILICES}

14

14

14

14

14

14

14

14

14

14

14

14

14

14

14

14

14

14

14

14

14

14

14

14

14

14

14

14

14

14

14

14

14

14

14

14

14

14

14

Geranium
Adiantum digitatum

Fteris semiserrata

1301

1301

16

Pteris triphylla

1301

16

FUMARIACEAE=FUMARICACEAE

Corydalis notrana

17

GENTINEAE $=$ GENTIANACEAE

Gentiana barbigera

807

Gentiana densiflora

805

11

Gentiana fimbricata

806

11

Gentiana flavescens

808

Lysianthus penduliflorus

815

Lysianthus vincaeflorus

817

Lysianthus viridifolius

816

Menyanthes pubiflora

819

Ophiorhiza undulata

814

Spigelia biflora

812

Spigelia ecalella

812

Spigelia elegans

810

Spigelia hedyotidea DC.

Spigelia marginata

813

811

Spigelia mexicana

811

Spigelia speciosa

810

Swertia gibeiflora

809

Swertia umbellata

GERANIACEAE

Geranium hernandesii DC. 147

Geranium mexicanum

147

3

potentillaefolium DC. $148 \quad 3$

GESSNERIEAE = GESSNERIACEAE Achymenes longiflora DC. $719 \quad 10$

Achymenes tenella DC. $\quad 720$

Conradia cuneifolia DC. 723

Gessneria chelonaeflora 724

Gessneria deppeana Schl. 722

Gessneria exogonia

717

Gessneria longiflora

719

Gessneria maculata DC.

721

Gessneria quaterniflora

Gessneria tenclea

722

720

Gessneria uniflora DC. 725

10

Gloxinia antirrhina DC
10

10

10

10

10

10

10

10

10

10 
GRAMINEAE

Andropogon

Maniscuris

Chloris

Chloris

Chloris

Chloris

Chloris

Chloris

Chloris

Chloris

Chloris

Ramirea

Hilaria

GROSSALARIACEAE = SAXIFRAGACEAE

Ribes fuchsioides

Ribes stamineum SM.

GUTTIFERAE

Mammea emarginata DC. 144

HYDROLEAE =HYDROPHYLACEAE

Hydrolea auriculata

Hydrolea decurrens

Hydrolea radians

Hydrolea rupincula

Hydrolea spinosa

Hydrolea scorpioides

Hydrolea tenella

Hydrolea violacea

Nama jamaicensis L.

Nama longiflora Choisy

Nama origanifolia Kunth.

Nama undulata Kunth.

Nama undulata Kunth.

Wigandia Kunthii Choisy

Wigandia

scorpioides Choisy

HYPERICACEAE

Hypericum

$$
\text { pauciflorum } \mathrm{DC} \text {. }
$$

HYPPOCRATEACEAE

Hippocratea acutiflora DC. 142

Hippocratea uniflora DC. 141

$\begin{array}{ll}1304 & 16 \\ 1309 & 16 \\ 1310 & 16 \\ 1320 & 16 \\ 1326 & 16 \\ 1328 & 16 \\ 1330 & 16 \\ 1331 & 16 \\ 1334 & 16 \\ 1335 & 16 \\ 1337 & 16 \\ 1312 & 16 \\ 1313 & 16\end{array}$

414

414

871

870

872

867

873

873

869

869

870

868

869

872

867

871

873

143

16

\section{HESPERIDACEAE}

16 Murraya simplicifolia

158

IRIDACEAE

Moraea quadriflora

1238

6 Sisyrhinchium

16 compressum

1232

Sisyrhinchium

gramineum

1237

Sisyrhinchium ixioides

Sisyrhinchium nanum

1236

16 Sisyrhinchium parviflorum

Tigrida mocronata

Tigrida obtusiflora

16 JUNCACEAE

Eriocaulon mexicanum 1283

\section{LABIATAE}

7 Calamintha macrostema

1012

Cedronella mexicana

1010

Dondosia integrifolia

1013

3 Dondisia serrata

1012

Fragosia capibellata

1015

Frajosia pedicellata

1014

Hyptis albida

1014

Leucas glabra

Leucas rubra

Melissa purpurascens

Molucella leonorus

1009

Monarda rosea

Nepeta mexicana

Plectranthus petiolaris

Plectranthus repens

Salvia affinis

Salvia agglutinans

Salvia ajujoides

Salvia amarisinna

Salvia ambigua

11 Salvia bulbosa

Salvia densiflora

1001

Salvia fastuosa

984

3 Salvia leptophylla

Salvia macrantha

991

3 Salvia melissafolia

998

3 Salvia mexicana 


\begin{tabular}{|c|c|c|c|c|}
\hline Salvia mociniana & 995 & 12 & Dalea acutifolia & 228 \\
\hline Salvia nepetoides & 993 & 12 & Dalea astragalina & 234 \\
\hline Salvia palafoxiana & 1000 & 12 & Dalea bicolor Willd. & 235 \\
\hline Salvia pauciflora & 1002 & 12 & Dalea citriodora Willd. & 230 \\
\hline Salvia secundiflora & 997 & 12 & Dalea flava & 237 \\
\hline Salvia spicata $N$ & 999 & 12 & Dalea flavo-rosea & 233 \\
\hline Salvia stricta & 986 & 12 & Dalea lateripes & 230 \\
\hline Salvia tuberosa & 987 & 12 & Dalea longipes & 231 \\
\hline Salvia ventricosa & 988 & 12 & Dalea lutea Willd. & 237 \\
\hline Scutellaria coccinea & 982 & 12 & Dalea mutabilis Willd. & 236 \\
\hline Scutellaria tuberosa $\mathrm{N}$. & 981 & 12 & Dalea melilotoides & 238 \\
\hline Teucrium mexicanum & 1006 & 12 & Dalea nutans Willd. & 238 \\
\hline \multicolumn{3}{|c|}{ LAURINEAE=LAURACEAE } & Dalea onobrychioides & 235 \\
\hline Laurus angustifolia & 1102 & 13 & Dalea pendulina & 232 \\
\hline Laurus coccinea & 1100 & 13 & Dalea procumbens & 229 \\
\hline Laurus pubiflora & 1101 & 13 & Dalea sericea Lag. & 231 \\
\hline Laurus viridis & 1104 & 13 & Detalostemon sessile & 239 \\
\hline Laurus trifida & 1103 & 13 & Dalea thouni & 239 \\
\hline Laurus uvifera & 1099* & 13 & Dalea tuberculata & 232 \\
\hline LEGUMINOSAE & & & Dalea unguicularis & 235 \\
\hline Aldinia glabra & 1175 & 14 & Darwinia anomala & 289 \\
\hline Abrus precatorius & $261 *$ & 5 & Drepanocarpus & \\
\hline Abrus arborescens & 261 & 5 & cyathiformis & 284 \\
\hline Acacia amentacea & 208 & 4 & Desmodium alamani & 268 \\
\hline Acacia esculenta & 209 & 4 & Desmodium alamani & 273 \\
\hline Acacia prosopoides & 210 & 4 & Desmodium infractum & 270 \\
\hline Amicia zygomeris & 275 & 5 & Desmodium stipulaceum & 269 \\
\hline Aeschynomene adscendens & 274 & 5 & Dolichos macrostachys & 243 \\
\hline Bahuinia leptopetala & 223 & 4 & Dolichos mutabilis & 241 \\
\hline Bahuinia spathacea & 224 & 4 & Dolichos palmatilobos DC. & 242 \\
\hline Caesalpinia biquadrijuga & 219 & 4 & Erythrina breviflora & 251 \\
\hline Caesalpinia elegantisima & 217 & 4 & Erythrina corallioides & 253 \\
\hline Caesalpinia exostemma & 218 & 4 & Erythrina divaricata & 256 \\
\hline Caesalpinia microphylla & 220 & 4 & Erythrina horrida & 252 \\
\hline Caesalpinia parviflora & 221 & 4 & Erythrina leptorhiza & 250 \\
\hline Canavalia ensiformis DC. & 278 & 5 & Erythrina longipes & 254 \\
\hline Cissolia spinosa & 281 & 5 & Erythrina patens & 255 \\
\hline Clitoria brasilensis & 1184 & 14 & Galactia radicata & 276 \\
\hline Clitoria plumieri & 240 & 4 & Galactia tuberosa & 277 \\
\hline Clitoria racemosa & 240 & 4 & Galega coronilloides & 263 \\
\hline Crotalaria procumbens & 227 & 4 & Glycine ensiformis & 241 \\
\hline Crotalaria setifera & 226 & 4 & Glycine geminiflora & 260 \\
\hline Crotalaria triantha & 225 & 4 & Glycine terniflora & 259 \\
\hline
\end{tabular}


Harpalyle formosa

249

Hedysarum geminiflorum 268

Hedysarum laxiflorum

271

Hedysarum mucronatum

Hedysarum procumbens

Hedwigia mexicana

Indigofera anil

Indigofera atropurpurea

Indigofera guatemalensis

Indigofera humilis

Indigofera tinctoria $\mathrm{B}$.

Ex. DC.

Inga houstoni

Inga quamochitl

Inga quaxiniquili

Inga latifolia Willd.

Inga trunca

Inga ungiscati Willd.

Inga vera

Marina densiflora

Mimosa geminata

Mimosa inga L.

Mimosa peregrina

Mucuna albida

Mucuna rutilans

Myrexylon acuminatum

Myrexylon ovale

Nissolia spinosa

Parkinsonia bipinnata

Phaseolus atropurpureus

Phaseolus auritus

Phaseolus bulbocastanum

Phaseolus linearis

Phaseolus pendulinus

Psoralea mutabilis Cav.

Pterocarpus ateleia

Pterocarpus crispatus

Pterocarpus falciformis

Pterocarpus orbiculatus

Requienia obtusiflora

Robina mexicana

Rudolphia breviflora

Rudolphia longiflora
273

272

204

264

266

265

267

265

206

212

213

214

214

212

213

118

205

213

207

278

279

286

287

281

215

244

247

246

248

245

236

288

282

285

283

1170

262

258

257
5

5

5

5

4

5

5

5

5

\section{5}

4

4

4

4

4

4

4

4

4

4

4

4

5

5

5

5

5

5
5

4

4

4

4

4

4

4

4

5

5

5
5

5

5

14

5

5
5

5
5

Rudolphia volubilis Willd.

Schrankia distachya

Securidaca virgata

257

Zornia reticulata

$289 *$

\section{LILIACEAE}

Agave angustifolia $\quad 1248$

Agave fibrillosa

1251

Alstroemeria enneantha 1247

Alstroemeria polyantha

1246

Anatis rigida $\quad 1267$

Bromelia brachystachya 1257

Crateis elegans

1264

Crinum hexanthum $\quad 1240$

Epipholea secundifolia

1254

Evadne geminiflora

1266

Gallesia citrina

1261

Heteranthera emarginata 1241

Heteranthera laxiflora 1242

Heteranthera obtusa $\quad 1243$

Heteranthera uniflora

1244

Hypoxis uniflora

1259

Lochnera bromeliaefolia

1263

Mancoa geminiflora 1266

Pitcairnia rupestris $\quad 1256$

Phalangium minimum 1258

Polianthes maculata Mart. 1251

Pontederia unifolia

1260

Tillandsia bracteosa

1253

Tillandsia marantaeflora

1252

Tillandsia recurvifolia

1255

Tillandsia secundifolia

1254

Tydea hypoxima

1269

Tydea ixina

1271

Tydea meleagrina

1268

Tydea tulipina

1270

Vibilia pubiflora

1272

Viguiera graminifolia

1262

1273

1245

Yucca mexicana

1189

LOASACEAAE

Loasa lyrata

Mentzelia hispida Willd. 357 
Mentzelia stipitata DC. $\quad 358$

Mentzelia zazale

\section{LOBELIACEAE}

Lobelia coccinea

Lobelia crispa Grah

Lobelia debilis $\mathrm{L}$.

Lobelia fulgens NBK

Lobelia hemisphaerica

A.DC.

Lobelia kunthiana DC.

Lobelia nana H.B.K.

Lobelia pumila

Lobelia pusilla DC.

Lobelia pyramidalis

Lobelia sempervirens N.S. 711

Lobelia sinuata

Lobelia spicata $\mathrm{N}$.

Lobelia unilateralis

Lobelia tenestralis Cav.

Lobelia tuberosa

Lobelia tupa Linn.

Lobelia xalapensi

Selliera ebracteata

\section{LORANTHACEAE}

Loranthus calyculatus DC. 449

Loranthus quachitli

Loranthus ramiflorus DC. 448

Rhizophora mangle

Rhizophora octandra

LYTHRACEAE

Bertolonia guieroides

Cuphea adscendens

Cuphea apanxaloa

Cuphea aquipetala Cav.

Cuphea cordifolia

Cuphea cyanea

Cuphea heptandra

Cuphea lanceolata

Cuphea micropetala

H.B.K.

Cuphea secundiflora

Cuphea strumosa Muc.

Cuphea tricolor
357

706

712

709

706

708

711

715

710

710

707

713

712

718

713

705

705

709

715

447

447

331

316

323

316

320

321

317

317

318

322

318

319
6

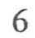

10

10

10

10

10

10

10

10

10

10

10

10

10

10

10

10

10

10

10

7

7

7

7

7

7

6

6

6

6

6

6

6

6

6

6

6

6
Cuphea tuxtlensis

Cuphea virgata

Ginoria flava

Grislea sessilifolia

Heimia syphilitica

Lafoensia mexicana DC. 314

Lythrum acinifolium

Lythrum alatum

Lythrum lanceolatum

Lythrum satureiaefolium

Nesaea salicifolia H.B.K.

Martia glaucina

Spirea opulifolia

MAGNOLIACEAE

Magnolia mexicana DC.

MALPHIGIACEAE

Banisteria brevipes DC. 129

Banisteria paniculata DC. 131

Banisteria ternata DC. $\quad 133$

Banisteria vitifolia DC. $\quad 132$

Byrsonima pulchra DC. 134

Galphimia glandulosa var ovalifolia DC.

140

Galphimia glandulosa

var oblongifolia DC.

139

Hiraea acuminata DC.

128

Hiraea cycloptera DC.

125

Hiraea macroptera DC. $\quad 130$

Hiraea mucronata DC. $\quad 124$

Hiraea podocarpa DC. $\quad 127$

Hiraea oxyota DC.

126

Malpighia

corymbifera DC. $\quad 137$

Malpighia emarginata DC. 135

Malpighia sessilifolia DC. 138

Malpighia urens DC. $\quad 136$

MALVACEAE

Alegria candida DC. $\quad 102$

Anoda acerifolia DC. 63

Carolinea fastuosa DC. $\quad 97$

Carolinea pompalis DC. 96

Cochlospermum

serratifolium DC. 
Eriodendron

aesculifolium DC.

94

Hibiscus acetosaefolius DC. 79

Hibiscus azanzae DC. $\quad 75$

Hibiscus bracteosus DC. $\quad 80$

Hibiscus columbinus DC. 78

Hibiscus cianogynus DC. 77

Hibiscus cylindriflorus DC. 76

Hibiscus fasciculatus DC. 85

Hibiscus flavidus DC. $\quad 87$

Hibiscus oxyphyllus DC. 86

Hibiscus pleurogenus DC. 81

Hibiscus pleurantherus DC. 84

Hibiscus tubiflorus DC. 83

Hibiscus uncinellus DC. $\quad 82$

Hibiscus virginicus $\quad 1184$

Ingenhouzia triloba DC. 101

Malva rosea DC. $\quad 58$

Malvaviscus candidus DC. 90

Malvoviscus penduliflorus DC. 91

Malvaviscus pentacarpus DC. 88

Malvaviscus trilobus DC. 89

Melochia rotundifolia DC. 93

Melochia corymbosa DC. 93*

Melochia conglobata DC. 92

Pavonia spinifex var. grandiflora DC.

Pavonia spinifex var. oblongifolia DC.

Myrodia verticillaris DC.

Myrodia ovata DC.

Myrodia turbinata SW.

Sida amplexifolia DC.

Sida brachystemon DC.

Sida carnea DC.

Sida malvaeflora DC.

Sida malvaviscus DC.

Sida melochioides DC.

Sida obcordata DC.

Sida oblicua DC.

Sida oxyphylla DC.

Sida palmata DC.

Sida quinqueloba
Sida rhomboidea DC.

62

Sida spinifex DC.

74

2

Urena grandiflora DC.

61

2 MELASTOMATACEAE

2 Heteronoma diversifolium 334

2 Lavoisiera albiflora

343

Melastoma

1184

2 Melastoma budleiaefolia 337

2 Melastoma carbata

M. y S.

Melastoma epilobia

341

Melastoma

epilobioides DC.

341

Melastoma

erythanthera M. y S.

338

Melastoma psychotria

340

Melastoma scabrosa

332

Melastoma solaniflora

Moc.\&Ses.

335

Miconia melastomoides

332

Rhexia inaqualifolia

Rhynchanthera

$$
\text { mexicana DC. }
$$

334

Tococa vesiculosa DC.

342

MELIACEAE

Cedrela angustifolia

Guarea brachystachya DC.

Styrax dependes DC.

Styrax racemosum DC.

Swietenia mexicana

Swietenia mahagoni L. $\quad 152$

Trichilia octandra

Trichilia pallida SW. 


\begin{tabular}{|c|c|c|c|c|}
\hline \multirow{2}{*}{\multicolumn{3}{|c|}{$\begin{array}{l}\text { Ruyschia mucronata } 1156 \\
\text { MYRSINEAE=MYRSINACEAE }\end{array}$}} & Fuchsia ovata & 367 \\
\hline & & & Fuchsia racemosa & 366 \\
\hline Ardisia bracteosa DC. & 740 & 10 & Gaura bracteata & 373 \\
\hline Ardisia capollina DC. & 741 & 10 & Gaura epilobia & 375 \\
\hline Manglilla sessiliflora & 738 & 10 & Gaura suffrutescens & 374 \\
\hline Myrsinci berberii & 739 & 10 & Isnardia mexicana & 360 \\
\hline Myrsine penduliflora & 739 & 10 & Jussiaea aquatilis & 369 \\
\hline MUSACEAE & & & Jussiaea erecta DC. & 371 \\
\hline Musa sapientum & 1229 & 15 & Jussiaea oxyphylla & 372 \\
\hline \multicolumn{3}{|c|}{ MYRTINEAE=MYRTALEAE } & Jussiaea peduncularis & 370 \\
\hline Lecythis nicaraguensis DC. & 345 & 6 & Jussiaea sessiliflora & 371 \\
\hline Myrtus mexicana & 344 & 6 & Mouriria mexicana & 361 \\
\hline NYCTAGINEAE & & & Oenothera latiflora & 376 \\
\hline Abronia acutiloba & 1085 & 13 & Oenothera tubifera & 377 \\
\hline Allionia affinis & 1075 & 13 & ORCHIDACEAE & \\
\hline Anagea cordifolia & 1083 & 13 & Cymbidium amomoides & 1203 \\
\hline Anagea glabra Moc. & 1084 & 13 & Cymbidium & \\
\hline Boerhaoria diffusa & $1086 *$ & 13 & anthericiflorum & 1210 \\
\hline Boerhaoria pentandra & 1086 & 13 & Cymbidium & \\
\hline \multicolumn{3}{|c|}{ Guamoclidion angulatum } & atropurpureum & 1208 \\
\hline Choisy & 1077 & 13 & Cymbidium leucopetalum & 1206 \\
\hline Mirabilis trianda & 1081 & 13 & Cymbidium limodoroides & 1195 \\
\hline Nyctago longissima & 1076 & 13 & Cymbidium limodoroides & $1195^{*}$ \\
\hline Nyctago racemosa & 1078 & 13 & Cymbidium maculatum & 1200 \\
\hline Nyctaginia capitata Choisy & 1082 & 13 & Cymbidium pallidum & 1205 \\
\hline Nyctago repanda & 1080 & 13 & Cymbidium pallens & 1201 \\
\hline Nictago sessilifolia & 1079 & 13 & Cymbidium palmifolium & 1196 \\
\hline Pisonia ambigua & 1089 & 13 & Cymbidium precatorium & 1204 \\
\hline Pisonia decandra & 1090 & 13 & Cymbidium pulcherrimum & 1207 \\
\hline Pisonia dichotoma & 1088 & 13 & Cymbidium repens & 1209 \\
\hline Pisonia volkameri & 1087 & 13 & Cymbidium secundiflorum & 1199 \\
\hline Vancoureria acuminata & 1082 & 13 & Cymbidium speciosum & 1194 \\
\hline \multicolumn{3}{|c|}{ ONAGRACEAE } & Cymbidium uniflorum & 1202 \\
\hline Cardionema multicaule DC & 381 & 6 & Cypripedium acuminatum & 1224 \\
\hline Epilobium mexicanum & 379 & 6 & Cypripedium turgidum & 1225 \\
\hline Epilobium undulatum & 378 & 6 & Dendrobium brevicornu & 1216 \\
\hline Fuchsia acuta & 364 & 6 & Dendrobium & \\
\hline Fuchsia alternans & 363 & 6 & pinguiculaeflorum & 1215 \\
\hline \multicolumn{3}{|l|}{ Fuchsia arborescens } & Epidendrum coccineum & 1191 \\
\hline Sims. (DC.) & 365 & 6 & Epidendrum fimbriatum & 1192 \\
\hline Fuchsia fulgens DC. & 362 & 6 & Epidendrum linearifolium & 1187 \\
\hline Fuchsia gracilis & 368 & 6 & Epidendrum luridum & 1190 \\
\hline Fuchsia hamellioides & 365 & 6 & & \\
\hline
\end{tabular}


Epidendrum

cinamomeum A. Rich. 1188

Epidendrum oxyphyllum 1189

Epidendrum polybulbon

A. Rich

1209

Epidendrum

radiatum Lindl.

1190

Epidendrum

rhizopharum Batem.

Erides mexicanum

1192

1211

Epidendrum

vitellinum Lindl.

1191

Govenia alba A. Rich. 1206

Habenaria monodactylis

Habenaria pentadactylis

Laelia albida Lindl.

Laelia majalus Lindi.

Masderallia mexicana

Malaxis leptostachya

Malcoxis ophioglossoides W.

Maloxis secundiflora

Neottia peregrina $\mathrm{N}$.

Neottia publiflora

Oncidium

Oncidium polyodon

Oncidium ramosum

Sexapias imbricata

Vanilla mexicana

OXALIDACEAE

Oxalis hernandesii DC.

Oxalis nudiflora DC.

Oxalis verticillata $\mathrm{DC}$.

PAPAVERACEAE

Bocconia frutescens var.cernua DC.

Bocconia integrifolia

Humb. et Bomp.

1222

1223

1201

1194

1212

1219

$1219^{*}$

1218

1220

1221

1189

1213

1214

1197

1193

149

150

151

Chelidonium multifidum DC. 16

PASSIFLORACEAE

Passiflora adenopoda DC. 32

Passiflora dictamo DC. 28

Passiflora serratistipula DC. 31

Passiflora trisetosa DC.

\section{4}

14

15

14

14

15

14

15

15

15

15

14

15

15

15

15

15

15

14

15

15

14

14

14

3

\section{.}

8
1
9

\section{PEDALINEAE =MARTYNEACEAE}

Ichnia verbenacea DC. 833

Maceria verbenacea

833

Martynia triloba

Sch. Cham.

832

Martynia unguis

832

833

11

11

Tamonea scabra

PERSONATAE=SCROPHULARIACEAE

Buchnera depressa

952

12

Buchnera eriniflora

955

Buchnera fimbliata

954

951

11

Buchnera multifida

Buchnera pinnatifida

1184

953

941

938

936

937

939

940

964

967

967*

946

948

947

949

958

960

961

959

974

956

Escobedia quinquenervia

Euphrosine angustifolia

968

970

Euphrosine erectiflora

Euphrosine multifida

972

969

Euphrosine nutans

Hermimeris ophrydiflora

Euphrosine pinnatifida

950

971

976

977

978
12

12

12

14

12

12

12

12

12

12

12

12

12

12

12

12

12

12

12

12

12

12

12

12

12

12

12

12

12

12

12

12

12 


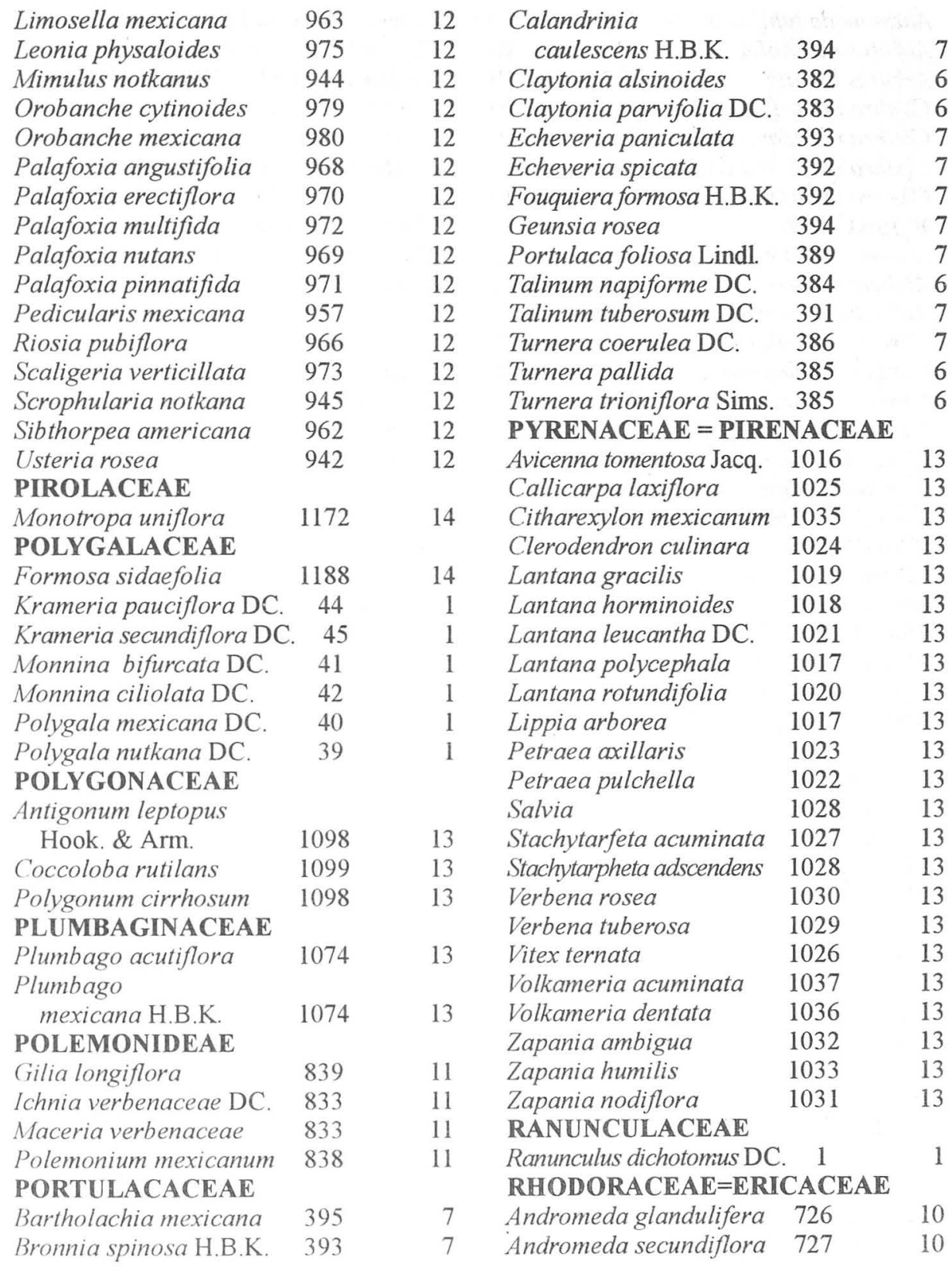




\begin{tabular}{|c|c|c|c|c|}
\hline Andromeda tubiflora & 728 & 10 & Catesbaea erecta DC. & 460 \\
\hline Arbutus ovalifolia & 729 & 10 & Cephaelis cyanorcarpa & 452 \\
\hline Arbutus bullata & 730 & 10 & Cephaelis tomentosa Willd. & 452 \\
\hline Clethra integrifolia DC. & 731 & 10 & Coffea rosea DC. & 479 \\
\hline Clethra mexicana DC. & 731 & 10 & Coutarea flavescens DC. & 459 \\
\hline Clethra mexicana DC. & 732 & 10 & Coutarea latiflora DC. & 458 \\
\hline Clethra ovalifolia & 732 & 10 & Diodia villosa DC. & 494 \\
\hline ROSACEAE & & & Ernodea mexicana & 478 \\
\hline Acaena secundiflora & 292 & 5 & Exostemma caribaum & 491 \\
\hline Alchemilla sessilis & 294 & 5 & Gardenia flava & 464 \\
\hline Alchemilla tormentillae & 295 & 5 & Genipa americana & 464 \\
\hline Cerasus capullin DC. & 306 & 5 & Genipa mexicana & 457 \\
\hline Cerasus ferruginea DC. & 307 & 5 & Genipa oblongifolia Ruiz & 457 \\
\hline Crataegus mexicana & 299 & 5 & Guettarda heptandra & 451 \\
\hline Geum cercocarpoides & 296 & 5 & Guettarda tetrandra & 450 \\
\hline Geum dryadoides & 297 & 5 & Hamellia patens var. & \\
\hline Hirtella albiflora & 305 & 5 & verticillata DC. & 489 \\
\hline Hirtella castanea & 304 & 5 & Hedyotis trichotoma & 500 \\
\hline Hirtella dodecandra & 302 & 5 & Higginsia corymbosa & 462 \\
\hline Hirtella oblongifolia & 303 & 5 & Hillia tetragona & 454 \\
\hline Hirtella octandra & 301 & 5 & Hillia tetrandra SW. & 455 \\
\hline Homalium senarium & 293 & 5 & Hillia tuxtlensis & 455 \\
\hline Hirtella triandra SW. & 305 & 5 & Houstonia mexicana var. & \\
\hline Lecostemon terniflorum & 311 & 5 & arenarioides & 499 \\
\hline Mespilus distyla & 300 & 5 & Houstonia mexicana var. & \\
\hline Nestleria inermis & 313 & 5 & sperguloides & $499 *$ \\
\hline Nestleria spinosa & 312 & 5 & Ixora pentandra & 485 \\
\hline Potentill lineariloba & 298 & 5 & Ixora spicata & 486 \\
\hline Prockia acuta & 308 & 5 & Ixora tenuiflora & 484 \\
\hline Prockia crucis Prodr. & 308 & 5 & Macrocnemum pallidum & 453 \\
\hline Prockia microstachya & 310 & 5 & Margaris barbigera DC. & 480 \\
\hline Prockia subcordata & 309 & 5 & Margaris nudiflora DC. & 481 \\
\hline Prunus canadensis L. & 306 & 5 & Mussanda breviflora & 475 \\
\hline Pyrus notkanus & $313 *$ & 5 & Mussanda nuda & 473 \\
\hline Pyrus notkanus & $315^{*}$ & 5 & Mussanda terniflora & 474 \\
\hline Rubus nutkanus & 291 & 5 & Nacibea racemosa & 461 \\
\hline Rubus trilobus & 290 & 5 & Oxyanthus triflorus & 467 \\
\hline Spiraea opulifolia & $313^{*}$ & 5 & Petesia odoratissima & 483 \\
\hline RUBIACEAE & & & Phaloe cobalonga & 468 \\
\hline Alperula cinanchica & 496 & 7 & Psychotria albiflora & 501 \\
\hline Bouvardia cordifolia DC. & 487 & 7 & Psychotria elegans & 502 \\
\hline Bouvardia quaternifolia DC. & 490 & 7 & Psychotria oblongata & 503 \\
\hline Carphalea pubiflora & 490 & 7 & Randia decussata & 470 \\
\hline
\end{tabular}


Randia diacantha 472

Randia echinocarpa DC. 469

Randia geminiflora

Randia latifolia

Richardia acutifolia

Richardsonia adscendens

Richardia ovalifolia

Rondeletia coccinea

Rondeletia odorata Jacq.

Rondeletia anomala

Rondoletia volubilis

Sabicea hirta SW.

Spermacoce asperuloides

Spermacoce galioides

Schradera lateriflora

Schradera polycephala

Stenostomum

dichotomum DC.

Viviana serrata

RUTACEAE

Guayacum acutifolium DC. 160

Plenckia grandiflora $\quad 161$

Plenckia parviflora $\quad 162$

Zygophyllum tridentatum DC.

SABIACEAE

Lavea parviflora

SAMYDEAE =FLACOURTIACEAE

Casearia dentata DC.

Casearia dubia DC.

Samyda macrocarpa DC.

Samyda rubra DC.

SAPINDACEAE

Cupania dentata DC. 123

Paullinia mexicana

Paullinia pteropoda DC.

Serjania mexicana

Willd. Ex DC.

Thouinia villosa DC.

SAPOTACEAE

Achras brevipes

Achras flavescens

Achras mespilus
1178

185

184

470

492

493

493

465

504

463

495

476

477

482

1173

183

182

120

121

120

122

746

747

751
496

7

7

7

\section{7}

14

3

3

3

3

3

14

3

3

3

3

3

2

2

2

2

2

2

2

10

10

10

\section{Achras microcarpa}

750

748

754

754

755

Bumelia dentiflora

$753 *$

752

$752 *$

753

753

756

756

757

742

Jacquinia fasciculata

Jacquinia racemosa DC.

Lavoisiera geminiflora

743

745

744

749

746

Lucuma capiri DC.

Lucuma salicifolia Kunth. 747

Macreightia

acapulcensis DC.
Pythagorea sapotalba

757

758

10

10

\section{SARMENTACEAE=DIOSCOREACEAE LILIACEAE VITIC}

$\begin{array}{lcc}\text { Cissus angulata Lam. } & 145 & 3 \\ \text { Cissus tuberosa DC. } & 146 & 3\end{array}$

\section{SAXIFRAGAE=SAXIFRAGACEAE}

Heuchera glabra Willd. $424 \quad 7$

Heuchera longipetala

423

Saxifraga notkana

422

422

Saxifraga stellaris

Tellima grandiflora S. Moc. 421

10

10

10

10

10

10

10

10

10

10

10

10

SASAMEAE = SCROPHULARIACEAE

$\begin{array}{lll}\text { Chelone integerrima } & 835 & 11 \\ \text { Chelone lincarifolia } & 836 & 11 \\ \text { Chelone mexicana } & 837 & 11 \\ \text { Chelone saponariaefolia } & 834 & 11\end{array}$

SMILACACEAE = SARMENTACEAE

\section{LILIACEAE}

Medeota notkana

1279

15

Smilax mecapatli

1276 
Smilax moranensis

Mart. \& Galeothi.

1276

Smilax oblongifolia

1275

Smilax oxyphylla

1277

Smilox schlechtendallii

1275

Smilax silvatica Kunth.

Smilax tuberosa

1276

1278

Tritillaria kamtschattensis 1279

SOLANEAE=SOLANACEAE

Acnistus aggregatus

915

Amphinome auriculata

923

Bouchetia erecta

921

Bouchetia procumbens Dun. 920

Brunfelsia mexicana

928

Cestrum leucocarpum

Cestrum nocturnum

911

911

Cestrum purpureum

912

Cestrum tomentosum

912

Crescentia alata H.B.K.

Crescentia pteropoda

Datura mateloides Dun

Jaborosa longiflora Dun.

Lycium aggregatum

926

926

919

Lycium quadrifidum Dun.

Nicotiana

ipomopsiflora Dun 909

Nicotiana longiflora Cav. 907

Nicotiana pilosa Dun. 908

Nicotiana plantaginea Dun. 910

Physalis cotztomatl

916

Physalis minutiflora

Physalis urceolata

918

917

Saracha contorta

$913 \mathrm{BI}$

Sinforosa flariloba

Sinforosa viridiloba

925

924

STERCULIACEAE

Sterculia oblongifolia DC. 106

Sterculia punctata DC. $\quad 107$

Theobroma angustifolia DC, 112

Theobroma guazuma L. $\quad 114$

Theobroma ovatifolia DC. 113

Waltheria brevipes DC. 110

Waltheria lanceolata DC. 109

\section{VIOLACEAE}

15 Calyptrion Berterii DC. 38

Hybanthus mexicanus

15

Gingins

37

1

Ionidium calceolarium

15

Gingins

35

15 Ionidium gracile DC.

36

15

12

12

12

12

12

12

12

12

12

12

12

12

12

12

12

12

Ionidium longifolium DC. 34

Sauvagesia geminiflora DC. 38*

Viola dichotoma $\mathrm{DC}$.

33

TEREBINTHACEAE=ANACARDIACEAE

Amyris acuminata

196

Amyris bipinnata

197

Amyris serrata DC.

196

Amyris tecomaca

195

Anacardium nicaraguense 188

Dodonaea emarginata

199

Comocladia acuminata

193

Elaphrium copalliferum

202

Elaphrium penicilatum

203

Fessonia dependens

194

Hedwigia mexicana

204

Picramnia tessonia

194

Ptelea podocarpa

200

Ptelea pentandra

201

Rhus filicina

189

12

12

Rhus heterophylla

191

Rhus pterocarpus

190

Rhus saxatilis

192

Rhus variifolium

191

Spathelia rhoifolia

190

Spondias myrobalanoides 198

4

4

4

4

4

4

4

4

4

4

4

4

4

4

Evelynia albiflora

1168

1168

4

4

4

4

4

4

4

12

12

Evelynia melioides

14

TERMINALIACEAE $=$ COMBRETACEAE

Gyrocarpus palmatifidus

1097

13

Terminalia leptostachya

1096

13

2 TILIACEAE

2 Corchorus

2

secundiflorus DC.

116

2

Helicteres

mexicana Kunth.

115

2

Helicteres rubiflora

115

.

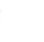

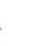

.

.

Miflora 115


Triumpheta acuminata

Triumpheta carnea

Triumpheta polyandria sp nov grandiflora

\section{TRANGULACEAE}

Albertinia shoefferioides DC. 168

Ceanothus carulens Lag. 176*

Ceanothus pauciflorus DC. 175

Celastrus mexicanus DC. 165

Colletia disperma DC. 173

Colletia multiflora DC. $\quad 174$

Govania stipularis DC. 178

Cyclostemon viridipes DC. 181

Henshawia tetrandra DC. 179

Rhamnus biniflorus DC. $\quad 171$

Rhamnus terniflorus DC. 170

Schaefferia mexicana DC. 167

Schaefferia racemosa DC. 169

Schaefferia

viridescens DC.

Staphylea paniculata

Torrentia distachya

Zyzyphus mexicanus

UMBELLIFERAE

Hacquetia bracteata

1169

Hydrocotyle grumosa DC. 426

Hydrocotyle natans

Hydrocotyle racemosa DC. 425
427

166

164

180

172

14

14

7
$2 \quad$ Hydrocotyle ranunculoides 428

2 Rethusa mexicana 430

Smyrnium mexicanum 429

2 URTICE=URTICACEAE

Cecropia penduliflora 1157

14

14

14

14

14

14

Urtica longifolia
UTRICULARINEAE=

LENTIBULARIACEAE

Pinguicola crenatiloba DC. $1071 \quad 13$

Pinguicola oblongiloba $1071 \quad 13$

Pinguicola obtusiloba DC. $1071 \quad 13$

Utricularia linearifolia 1072

13

VALERIANACEAE

3 Valeriana capitellata 506

Valeriana ceratophylla

H.B.K.

507

Valeriana dichotoma $\quad 507$

Valeriana scandens L. $\quad 505$

Valeriana volubilis

505

\section{ZANTHOXYLACEAE}

Zanthoxylum

aromaticum Willd.

186

4

Zanthoxylum mexicanum 186

Zanthoxylum pentanome 187

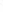

\section{4}

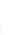

